(1)

CrossMark

\title{
Roles of HIF1 and HIF2 in pulmonary hypertension: it all depends on the context
}

\author{
Gregory B. Waypa and Paul T. Schumacker
}

Affiliation: Dept of Pediatrics, Northwestern University Feinberg School of Medicine, Anne and Robert H. Lurie Children's Hospital, Chicago, IL, USA.

Correspondence: Paul T. Schumacker, Northwestern University, 305 East Superior St, SQBRC 4-526, Chicago, IL 60611-3008, USA. E-mail: p-schumacker@northwestern.edu

@ERSpublications

HIF1 and HIF2 can play complementary, opposing or unrelated roles in mediating the response to low oxygen levels in different cell types. This study shows HIF2 plays a dominant role in mediating the development of PH in response to environmental hypoxia. http://bit.ly/2Cy46sR

Cite this article as: Waypa GB, Schumacker PT. Roles of HIF1 and HIF2 in pulmonary hypertension: it all depends on the context. Eur Respir J 2019; 54: 1901929 [https://doi.org/10.1183/13993003.01929-2019].

Pulmonary hypertension $(\mathrm{PH})$ is a diverse collection of vascular disorders that cause remodelling of small pulmonary arteries, resulting in increases in pulmonary vascular resistance and pulmonary arterial pressure. The World Health Organization classifies these disorders into five groups. Group III includes pulmonary hypertension associated with hypoxic lung disorders such as COPD. While not everyone with COPD develops pulmonary hypertension, those who do are more likely to experience acute exacerbations, hospitalisations, and poorer outcomes. Because alveolar hypoxia is a key element driving this response, investigators have studied how chronic hypoxia contributes to the development of $\mathrm{PH}$.

Cells respond to hypoxia by expressing genes that protect against the consequences of oxygen deprivation, augment/restore tissue oxygen delivery, and shift metabolism toward pathways that conserve cellular homeostasis. The most important transcription factors driving these responses are the hypoxia-inducible factors 1 and 2 (HIF1 and HIF2) [1]. These are controlled principally by regulating the abundance of their respective alpha subunits in accordance with the cellular level of oxygen. Under normoxic conditions, HIF $1 \alpha$ and HIF $2 \alpha$ are rapidly degraded, whereas during hypoxia their abundance increases, allowing them to heterodimerise with the HIF1 $\beta$ subunit, translocate to the nucleus, and activate transcriptional responses $[2,3]$.

To study the roles of HIF1 and HIF2 in PH, investigators have employed genetic or pharmacological manipulations to inhibit or activate their responses. Not surprisingly, germline deletion of HIF1 $\alpha$ is embryonic lethal, while deletion of HIF $2 \alpha$ produces lethality in some genetic backgrounds and a severe phenotype in others. To circumvent this, SHIMODA et al. [4] studied mice that were heterozygous for HIF $1 \alpha$ and found that the pulmonary response to chronic hypoxia was significantly attenuated. Others have used smooth muscle-specific HIF1 $\alpha$ deletion in adult mice, which attenuates (but does not abolish) the PH and vascular remodelling responses to environmental hypoxia [5]. Collectively, those studies and others [6, 7] suggested that HIF1 activation contributes to the vascular remodelling response and PH. However, others have reported that HIF1 activity lowers pulmonary vascular tone by decreasing myosin light-chain phosphorylation, which argues for a protective role of HIF against $\mathrm{PH}$ [8]. 


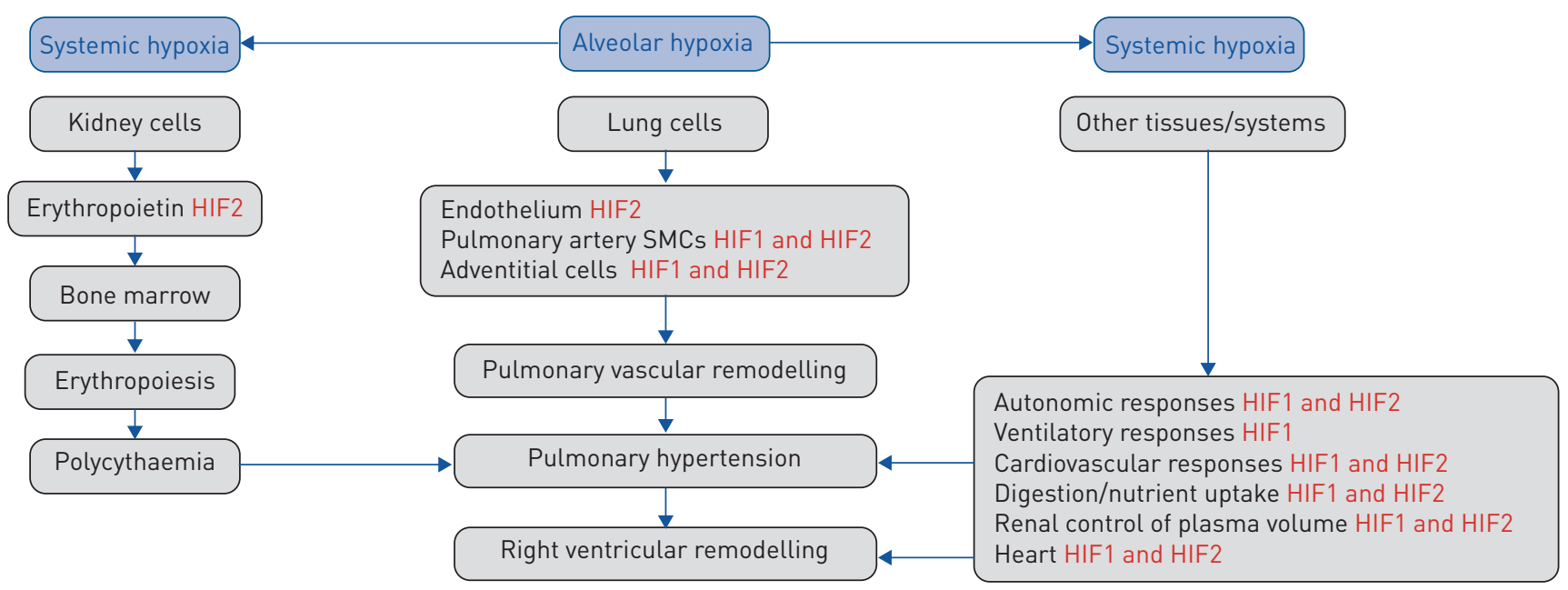

FIGURE 1 Hypoxia-inducible factors 1 and 2 (HIF1 and HIF2) play important roles in regulating transcriptional responses affecting the development of pulmonary hypertension. Alveolar hypoxia acts directly on lung epithelium, endothelium, vascular smooth muscle and adventitial cells, where HIF1 and HIF2 drive cell-specific responses that orchestrate the development of pulmonary hypertension. Alveolar hypoxia also leads to systemic hypoxaemia, which activates HIF-dependent responses that influence the degree of pulmonary hypertension. For example, renal hypoxia drives erythropoietin via HIF2 activation; the resulting increase in haematocrit augments pulmonary arterial pressure. HIF-dependent transcription in the carotid body affects the ventilatory response to hypoxia, which affects alveolar and arterial oxygen tension. Systemic hypoxaemia lowers right ventricular arterial oxygen tension, potentially activating HIF-dependent responses that affect cardiac remodelling. Importantly, HIF1 and HIF2 control distinct transcriptional profiles that are unique to each cell type. SMCs: smooth muscle cells.

Understanding the role of HIF in PH requires consideration of the diverse effects of HIF in different cell types (figure 1). For example, HIF in endothelial cells can help to drive proliferation and survival, and the expression of factors such as endothelin-1 that promote proliferation and constriction of cells in the medial layer of the pulmonary arteries. Moreover, systemic hypoxaemia triggers the expression of erythropoietin, causing an increase in haematocrit (which augments pulmonary vascular resistance). Hypoxia in the right ventricle may also cause HIF-dependent effects on remodelling that occur independently from those caused by increases in pulmonary artery pressure. We also know that HIF1 and HIF2 regulate overlapping but distinct genes, such that each one activates (or inactivates) a different gene set depending on the cell type [9]. These observations lead to the conclusion that understanding the roles of HIF1 and HIF2 in the pulmonary vascular response to chronic hypoxia requires understanding of the cellular context.

Recognising this, Hu et al. [10] set out to understand the roles of HIF1 and HIF2 in PH. As reported in this issue of the European Respiratory Journal, they first deleted HIF1 $\alpha$ systemically in adult mice using Ubc-Cre-ERT2, which causes widespread deletion in cells and tissues. Surprisingly, and in contrast to previous work, they found no attenuation of right ventricular systemic pressure (RVSP, a surrogate of pulmonary arterial pressure) or right ventricle hypertrophy after chronic hypobaric hypoxia. Using the same approach to delete HIF2 $\alpha$, they found that the mice all died after $\sim 3$ weeks of hypoxia, possibly due to lack of a sufficient autonomic sympathetic response. Mice with systemic deletion of only one HIF $2 \alpha$ allele survived in hypoxia and exhibited an attenuation of right ventricle remodelling and right ventricle weight, but no decrease in RVSP. Next, they turned to antisense oligonucleotides to suppress HIF2 $\alpha$, and found that survival was preserved while haematocrit, RVSP, Fulton's index and catecholamine responses during chronic hypoxia were attenuated. Antisense nucleotides against HIF1 $\alpha$ had no effect on the development of PH, underscoring their Ubc-Cre-ERT2-HIF1 $\alpha$ findings. Collectively, their studies identify an important role for HIF2, but not HIF1, in the pulmonary vascular response to chronic hypoxia. Finally, they utilised a HIF2-specific inhibitor, PT2567, in rats and found that mean pulmonary artery pressures, right ventricle remodelling and pulmonary artery wall remodelling responses were attenuated while the abundance of inflammatory cells, proliferating cells and expression of tenascin c (a glycoprotein lined to extracellular matrix remodelling and proliferation) were decreased. These findings were consistent with their murine studies and support the importance of HIF2 in diverse cell types in the pulmonary artery wall.

To their credit, Hu et al. [10] strengthened their in vivo analysis by assessing HIF-dependent gene expression patterns in vascular cells cultured under normoxic versus hypoxic conditions. Those results, along with siRNA knockdown studies and conditioned media experiments, underscored the importance of HIF2-dependent gene responses in endothelial cells in controlling the response to hypoxia. But which cells 
are responsible for driving the HIF2-dependent $\mathrm{PH}$ phenotype? To answer this, they used an endothelial cell knockout of HIF $2 \alpha$ in mice, and found that this essentially abolished the pulmonary vascular response to chronic hypoxia, along with the RVSP response and right ventricle remodelling. These studies identify the importance of HIF2 in the endothelium in driving the pulmonary artery responses to chronic hypoxia.

As is frequently the case with interesting studies, the report by $\mathrm{Hu}$ et al. [10] answers some questions while it raises others. HIF2 regulates erythropoietin expression in the kidney [11], so it is not surprising that the systemic antisense knockdown suppressed the polycythaemia that develops during chronic hypoxia. To what extent is this responsible for attenuating the increase in pulmonary artery pressure and decreasing right ventricle remodelling? Systemic administration of PT2567 should have also abolished the chronic hypoxia-induced polycythaemia, although haematocrit levels in that study were not reported. It seems possible that the failure to increase blood viscosity in HIF2-inhibited mice could lessen the development of $\mathrm{PH}$, thereby suppressing right ventricular remodelling. In addition, while they show that the expression of many genes associated with $\mathrm{PH}$ is affected by a loss of HIF2, the roles of those genes in mediating the $\mathrm{PH}$ response to chronic hypoxia will need to be determined in future studies.

Interestingly, the endothelial cell knockout of HIF2 virtually abolished the $\mathrm{PH}$ and right ventricle responses to chronic hypoxia, yet this had no effect on the increase in haematocrit. Previously, endothelial PHD2 deletion during embryonic development was shown to cause a severe HIF2-dependent PH response in normoxic mature mice [12]. However, PHD2 deletion in adult mice does not produce the same phenotype. The study by Hu et al. [10] shows a more dramatic effect of embryonic endothelial HIF2 knockout (compared with PT2567 or the antisense oligonucleotide interventions in adult mice) in terms of blocking the response to chronic hypoxia. Hence, HIF2 may play an important role in the developing lung, in terms of setting the context for later development of $\mathrm{PH}$. In either case, the findings by $\mathrm{Hu}$ et al. [10] move the field forward by identifying the critical importance of endothelial HIF2 in mediating the $\mathrm{PH}$ response to alveolar hypoxia.

Conflict of interest: G.B. Waypa has nothing to disclose. P.T. Schumacker has nothing to disclose.

Support statement: This work was supported by the National Institutes of Health (HL109478, HL122062 and HL35440). Funding information for this article has been deposited with the Crossref Funder Registry.

\section{References}

1 Semenza GL. Oxygen homeostasis. Wiley Interdiscip Rev Syst Biol Med 2010; 2: 336-361.

2 Ivan $\mathrm{M}$, Kondo $\mathrm{K}$, Yang $\mathrm{H}$, et al. HIFo targeted for VHL-mediated destruction by proline hydroxylation: implications for $\mathrm{O}_{2}$ sensing. Science 2001; 292: 464-468.

3 Jaakkola P, Mole DR, Tian YM, et al. Targeting of HIF- $\alpha$ to the von Hippel-Lindau ubiquitylation complex by $\mathrm{O}_{2}$-regulated prolyl hydroxylation. Science 2001; 292: 468-472.

4 Shimoda LA, Manalo DJ, Sham JSK, et al. Partial HIF-1 $\alpha$ deficiency impairs pulmonary arterial myocyte electrophysiological responses to hypoxia. Am J Physiol Lung Cell Mol Physiol 2001; 281: L202-L208.

5 Ball MK, Waypa GB, Mungai PT, et al. Regulation of hypoxia-induced pulmonary hypertension by vascular smooth muscle hypoxia-inducible factor-1 $\alpha$. Am J Respir Crit Care Med 2014; 189: 314-324.

6 Shimoda LA. Let's talk about sex: a novel mechanism by which estrogen receptor $\beta$ limits hypoxia-inducible factor expression in pulmonary endothelial cells. Am J Respir Cell Mol Biol 2018; 59: 11-12.

7 Frump AL, Selej M, Wood JA, et al. Hypoxia upregulates estrogen receptor $\beta$ in pulmonary artery endothelial cells in a HIF-1 $\alpha$-dependent manner. Am J Respir Cell Mol Biol 2018; 59: 114-126.

8 Kim YM, Barnes EA, Alvira CM, et al. Hypoxia-inducible factor-1 $\alpha$ in pulmonary artery smooth muscle cells lowers vascular tone by decreasing myosin light chain phosphorylation. Circ Res 2013; 112: 1230-1233.

9 Loboda A, Jozkowicz A, Dulak J. HIF-1 versus HIF-2 - is one more important than the other? Vascul Pharmacol 2012; 56: 245-251.

$10 \mathrm{Hu}$ C-J, Poth JM, Zhang H, et al. Suppression of HIF2 signalling attenuates the initiation of hypoxia-induced pulmonary hypertension. Eur Respir J 2019; 54: 1900378.

11 Rankin EB, Biju MP, Liu Q, et al. Hypoxia-inducible factor-2 (HIF-2) regulates hepatic erythropoietin in vivo. J Clin Invest 2007; 117: 1068-1077.

12 Dai Z, Li M, Wharton J, et al. Prolyl-4 hydroxylase 2 (PHD2) deficiency in endothelial cells and hematopoietic cells induces obliterative vascular remodeling and severe pulmonary arterial hypertension in mice and humans through hypoxia-inducible factor-2 $\alpha$. Circulation 2016; 133: 2447-2458. 